\title{
Índices dietéticos na avaliação da qualidade global da dieta
}

\section{Dietetic indexes for the assessment of overall diet quality}

Ana Maria CERVATO ${ }^{1}$

Viviane Laudelino VIEIRA2

\section{RES U M O}

A alimentação pode atuar tanto na prevenção de certas doenças como no tratamento de outras. A análise do consumo alimentar fornece subsídios para avaliação das necessidades nutricionais e para promoção de programas de educação nutricional. Poucos estudos têm sido realizados com o objetivo de se fazer a avaliação global da dieta. Os índices dietéticos estão sendo estudados como uma alternativa para realizar esta avaliação. Este trabalho tem por objetivo apresentar os seguintes índices dietéticos: Índice de Nutrientes, Escore de Variedade da Dieta, Escore de Diversidade da Dieta, Índice de Qualidade da Dieta, Índice de Alimentação Saudável e Índice de Qualidade da Dieta Revisado. Observou-se que os índices possuem fundamental importância ao refletir a situação de diversos componentes da dieta em uma única variável.

Termos de indexação: avaliação nutricional, consumo de alimentos, saúde pública, dieta, nutrição.

\section{A B S T R A C T}

Feeding can act in the prevention of some diseases as well as in the treatment of others. The analysis of food consumption provides information to assess nutritional needs and improve nutrition education programs. Few studies have been carried out in order to perform a global dietary assessment. The dietary indexes have been studied as an alternative to perform this assessment. The objective of this paper is to show some dietary indexes: Nutrient Index, Diet Variety Score, Diet Diversity Score, Diet Quality Index, Healthy Eating Index and Diet Quality Index Revised. These indexes have fundamental importance in the summarization of distinct dietary components in a single variable.

Index terms: nutrition assessment, food consumption, public health, diet, nutrition.

\footnotetext{
${ }^{1}$ Departamento de Nutrição, Faculdade de Saúde Pública, Universidade de São Paulo. Av. Dr. Arnaldo, 715, Cerqueira César, 01246-904, São Paulo, SP, Brasil. Correspondência para/Correspondenceto: A.M.CERVATO.

2 Graduanda, Faculdade de Saúde Pública, Universidade de São Paulo. Bolsista de Iniciação Científica do CNPq.
} 


\section{N T R O D U Ç Ã O}

A alimentação nutricionalmente adequada pode atuar tanto na prevenção como no tratamento de doenças. Assim, tem-se verificado cada vez mais a importância da avaliação dos hábitos alimentares da população. Pela análise dietética, as necessidades nutricionais das pessoas podem ser identificadas e programas de educação nutricional podem ser implementados, a fim de se proporcionar melhores condições de saúde para a população ${ }^{1,2}$.

Historicamente, guias alimentares foram desenvolvidos como instrumento que determina quais escolhas alimentares são necessárias para se alcançarem as necessidades nutricionais por meio da dieta ${ }^{3}$.

No tocante aos hábitos alimentares, poucos estudos têm sido realizados com o objetivo de se fazer a avaliação global da dieta ${ }^{4,5}$. Os índices dietéticos estão sendo estudados como uma alternativa para realizar esta avaliação².

0 índice dietético consiste em um método de análise da alimentação de indivíduos com a finalidade de determinar a qualidade da dieta através de um ou mais parâmetros, tais como: ingestão adequada de nutrientes, número de porções consumidas de cada grupo de alimentos (leite e substitutos, carnes, cereais, frutas e hortaliças), quantidade de diferentes gêneros alimentícios presentes na dieta ${ }^{6}$.

Portanto, este trabalho foi realizado com o objetivo de abordar a análise global da dieta, a partir de buscas bibliográficas realizadas em bancos de dados, como o M EDLINE e o DEDALUS, visando apresentar alguns diferentes índices para avaliação de qualidade.

\section{Índices para a avaliação da dieta}

O conceito de qualidade da dieta evoluiu com o tempo. Antigamente, era dada maior importância na prevenção da deficiência de nutrientes. As dietas que supriam as recomendações para energia e para os nutrientes essenciais conhecidos até então eram consideradas adequadas. Com o reconhecimento da idéia de associação de fatores dietéticos à prevenção e promoção de doenças crônicas, características dietéticas associadas à redução do risco dessas doenças foram incluídas à qualidade da dieta.

Nas últimas décadas, principalmente nos Estados Unidos, vários índices foram criados a partir de guias dietéticos, em especial do guia

Quadro 1. Descrição de características dos índices de avaliação da qualidade de dieta.

\begin{tabular}{|c|c|c|c|}
\hline Índice & Inquérito & Variáveis & Amostra \\
\hline $\mathrm{IN}^{1}$ & $\begin{array}{l}\text { Questionário de consumo de } \\
\text { alimentos }\end{array}$ & $\begin{array}{l}\text { A limentos ricos em proteínas, leite, frutas/horta- } \\
\text { liças e cereais }\end{array}$ & 3318 participantes do NFCS ${ }^{2}$ \\
\hline $\mathrm{EVD}^{3}$ & $\begin{array}{l}\text { Recordatório de } 24 \mathrm{~h} \text { e registro } \\
\text { de alimentos }\end{array}$ & Número de alimentos consumidos & 24 jovens e 24 idosos \\
\hline $\mathrm{EDD}^{4}$ & Histórico dietético & Número de grupos de alimentos consumidos & 837 adultos \\
\hline $\mathrm{IQD}^{5}$ & $\begin{array}{l}\text { Recordatório de } 24 \mathrm{~h} \text { e registro } \\
\text { de alimentos }\end{array}$ & $\begin{array}{l}\text { Gorduras total e saturada, colesterol, fruta/hor- } \\
\text { taliça, cereais/leguminosas, proteína, sódio e } \\
\text { cálcio }\end{array}$ & $\begin{array}{l}5484 \text { adultos participantes do } \\
\text { NFCS }\end{array}$ \\
\hline IAS $^{6}$ & $\begin{array}{l}\text { Recordatório de } 24 \mathrm{~h} \text { e registro } \\
\text { de alimentos }\end{array}$ & $\begin{array}{l}\text { Cereais, hortaliças, frutas, leite, carne, gorduras } \\
\text { total e saturada, colesterol, sódio e variedade }\end{array}$ & $\begin{array}{l}3997 \text { participantes do CSFII }{ }^{7} \text { acima } \\
\text { de } 2 \text { anos }\end{array}$ \\
\hline IQD-R $R^{8}$ & Recordatório de $24 \mathrm{~h}$ & $\begin{array}{l}\text { Gorduras total e saturada, colesterol, frutas } \\
\text { hortaliças, cereais, cálcio, ferro, diversidade e } \\
\text { moderação }\end{array}$ & 3202 adultos participantes do CSFII \\
\hline
\end{tabular}

(1) IN = Índice de Nutrientes (Jenkins \& Guthrie, 1984); ${ }^{8}$ NFCS=Nationwide Food Consumption Survey; ${ }^{(3)}$ EVD=Escore de Variedade da Dieta (Krebs-Smith et al., 1987) ; ${ }^{(4)}$ EDD=Escore de Diversidade da Dieta (Drewnowski et al., 1996) ${ }^{10}$; ${ }^{(5)} \mid \mathrm{QD}=$ =́ndice de Qualidade da Dieta (Patterson et al., 1994) ${ }^{6} ;{ }^{(6)}$ IAS=Índice de Alimentação Saudável (Bowman et al., 1998) ${ }^{15} ;{ }^{(7)}$ CSFII=Continuing Surve of Food Intakes by Individuals; ${ }^{(8)}$ IQD-R=índice de Qualidade da Dieta Revisado (Haines et al., 1999) ${ }^{13}$. 
dietético para americanos e da pirâmide de alimentos ${ }^{7}$.

A nalisando-se as características de diversos índices de avaliação da dieta (Quadro 1), observa-se a evolução do conceito de qualidade, com a diminuição da preocupação em atingir adequação para inúmeros nutrientes e o aumento do interesse em englobar fatores dietéticos ligados à prevenção das doenças mais prevalentes na atualidade 5 . Para uma melhor compreensão dessa evolução, cada um dos índices será descrito separadamente.

\section{Índice de nutrientes (IN)}

Foi criado em 1984 por Jenkins e Guthrie, a partir do estudo de dietas de três dias de uma amostra da população. Devido à quantidade crescente de nutrientes importantes para serem analisados, segundo as recomendações dietéticas para os americanos, verificou-se a necessidade de se reduzir o número de indicadores do estado nutricional. Assim, o objetivo desse estudo foi identificar um número menor de nutrientes, geralmente usado para avaliar a dieta e examinar a relação desses com outros micronutrientes.

Os dados foram obtidos por meio de questionário de freqüência de consumo de alimentos de uma amostra da população adulta (3318 indivíduos) participante do Nationwide Food Consumption Survey (NFCS), entre os anos de 1977 e 1978.

A análise foi feita pela quantidade de alguns nutrientes ingeridos. Através dos valores encontrados para esses nutrientes, pôde-se estimar se a ingestão dos demais estava adequada. Os nutrientes foram organizados em quatro grupos. 0 grupo 1 contém alimentos fontes de proteínas, pressupondo que estes também forneçam vitamina $B_{6}$, ferro, niacina e zinco. 0 grupo 2 contém o leite e seus substitutos, indicando a ocorrência de ingestão do cálcio, da riboflavina, do fósforo, da vitamina $B_{12}$ e do magnésio. 0 grupo 3 contém as frutas e hortaliças, relacionando-as ao consumo da folacina (folato), vitamina A e vitamina C. Por fim, o grupo 4 contém os cereais, pressupondo o consumo da tiamina, além dos carboidratos ${ }^{8}$.

\section{Escore da variedade da dieta (EVD)}

Foi desenvolvido em 1987 por Krebs-Smith et al. (1987) ${ }^{9}$, considerando o fato de a escolha de alimentos variados dentro dos grupos de alimentos e entre eles promover uma melhora na alimentação, porque as vitaminas, os minerais e outros micronutrientes são obtidos em quantidades ideais para favorecer a saúde.

O grupo populacional estudado constituiu-se de 24 jovens entre 20 e 30 anos e 24 idosos entre 60 e 75 anos, sendo 24 homens e 24 mulheres. Os dados foram coletados a partir de um recordatório de 24 horas e de registro de alimentos de 14 dias consecutivos. O EVD foi definido como o número de diferentes itens de alimentos consumidos em um determinado período.

Seu objetivo foi desenvolver uma nova medida de variedade da dieta e englobá-la a outras medidas de qualidade da dieta, já que as avaliações dietéticas existentes até então davam muito enfoque para a quantidade energética e de nutrientes ingeridos e pouco para a variedade da dieta escolhida ${ }^{7}$. No entanto, vários estudos têm assumido a importância da diversidade, qualidade e variedade da dieta ${ }^{3,10}$. 0 guia dietético para americanos ${ }^{11}$ e a pirâmide de alimentos, do Departamento de Agricultura dos Estados Unidos $^{12}$, também vêm destacando há muito tempo a importância do consumo de uma dieta variada.

\section{Escore da diversidade da dieta (EDD)}

Foi criado por Drewnowski et al., através de estudo conduzido entre 1988 e 1989 por membros do Institut Scientifique et Technique de I'Alimentation, em Paris, França. Para a amostra do estudo, selecionaram-se 837 adultos (361 
homens e 476 mulheres). Foi usado na avaliação da dieta o método do histórico dietético, no qual os entrevistadores questionavam sobre hábitos alimentares freqüentes nos últimos seis meses. Esse índice quantifica o número de diferentes grupos de alimentos consumidos diariamente pela amostra da população. Esses grupos abrangem: leite e substitutos, carne, cereais, frutas e hortaliças ${ }^{10}$.

Os escores das dietas de cada participante são calculados considerando uma lista pré-determinada de 73 alimentos divididos nos grupos de alimentos acima citados. 0 escore máximo é 5, com um ponto dado para cada grupo de alimento consumido. Nesse estudo, não foram analisadas as ingestões de bebidas alcoólicas, gorduras e doces ${ }^{10}$.

\section{Índice de qualidade da dieta (IQD)}

Foi desenvolvido em 1994 por Kant, com o objetivo de criar um instrumento de medida da qualidade global da dieta, que refletisse um gradiente de risco para muitas doenças crônicas relacionadas com a alimentação $0^{6,3}$. Além disso, pretendia identificar pessoas com dietas de alta qualidade, baseando-se na possibilidade de se alcançar as necessidades nutricionais e diminuir a ingestão de gorduras total e saturada. Este índice é baseado na importância de determinados nutrientes e nas recomendações dietéticas do Food and Nutrition Board².

Indivíduos com o registro de três dias da dieta (um recordatório de 24 horas e dois registros de alimentos consumidos em dois dias distintos) foram estudados para se captar melhor o padrão básico da dieta e para limitar a variação entre indivíduos. A amostra foi composta por 5484 americanos maiores de 21 anos, participantes do Nationwide Food Consumption Survey de 1987 e 1988.

O IQD incorporou um esquema no qual três elementos da dieta (gordura total, gordura saturada e colesterol) receberam maior destaque quando comparados a outros nutrientes. 0 quarto componente refere-se ao número de porções de frutas e hortaliças, enquanto 0 quinto indica 0 número de porções de cereais e leguminosas. Os três últimos elementos analisam as ingestões de proteína, sódio e cálcio. Indivíduos que tiveram consumo adequado de cada indicador receberam nota zero, enquanto aqueles com ingestões muito diferentes das adequadas receberam do is pontos. A pontuação final foi a soma dos oito indicadores, totalizando um mínimo de zero (dieta excelente) e um máximo de 16 (dieta ruim). Os resultados do estudo indicaram que o IQD identifica a qualidade global da alimentação por incorporar vários fatores dietéticos ${ }^{6}$.

\section{Índice de alimentação saudável (IAS)}

Foi criado em 1995 pelo Departamento de Agricultura dos Estados Unidos, através de um estudo cujo objetivo foi construir um índice de qualidade global da dieta que incorpora as necessidades nutricionais e os guias dietéticos para consumidores norte-americanos em uma só medida ${ }^{3}$.

A amostra da população incluía 3997 pessoas maiores de dois anos, participantes do Continuing Survey of Food Intakes by Individuals (CSFII), de 1989 e 1990. Ele foi desenvolvido a partir de informações atualizadas disponíveis até a sua publicação, incluindo o Guia Dietético para Americanos ${ }^{14}$.

É uma medida simples e sumária da qualidade da dieta, a qual pode ser usada para monitorar as mudanças nos padrões de consumo e também para servir como instrumento útil na educação nutricional e promoção da saúde. As informações dietéticas são analisadas em um recordatório de 24 horas e um registro de dois dias de alimentos.

O IAS é constituído por dez componentes que estão baseados em diferentes aspectos de uma dieta saudável. Para cada componente, há uma pontuação variando de zero a 10. Assim, o 
índice varia de zero a 100. Os componentes de 1 a 5 medem a dieta conforme as recomendações de porções da Pirâmide de Alimentos para os cinco grupos principais: cereais, hortaliças, frutas, leite e carnes. 0 componente 6 é baseado no consumo de gordura como uma porcentagem da quantidade de energia total fornecida pela dieta. 0 componente 7 é baseado no consumo de gordura saturada como uma porcentagem da quantidade de energia total fornecida pela dieta. Os componentes 8 e 9 estão baseados nas ingestões de colesterol e sódio. Por fim, o componente 10 é baseado na variedade dietética de uma pessoa ${ }^{14,15}$.

\section{Índice de qualidade da dieta revisado (IQD-R)}

A revisão foi feita em 1999. Desde a criação do IQD original, as recomendações dietéticas têm mudado. Surgiram a pirâmide de alimentos e o guia dietético para americanos, ambos com novas propostas. Os objetivos desse estudo foram revisar o IQD, refletindo sobre as recomendações dietéticas existentes até então, e avaliar os critérios nutricionais nos quais o índice está baseado. Ele também visa adotar um método de medidas de porções de frutas, hortaliças e cereais de modo mais consistente ${ }^{13}$.

A dieta foi avaliada a partir da média de do is recordatórios de 24 horas de cada indivíduo da amostra, com intervalo de 10 dias entre eles. Dados de consumo foram obtidos de pessoas acima de 18 anos que participaram do Continuing Survey of Food Intakes by Individuals, de 1994. A amostra era constituída por 3202 pessoas ${ }^{13}$.

Três novos conceitos entram em conjunto com a distribuição de recomendações de macronutrientes: variação, moderação e proporcionalidade. A proporcionalidade se refere à recomendação de se consumir mais alimentos de determinados grupos e menos de outros. A moderação reflete 0 princípio de limitar 0 consumo de certos constituintes de alguns alimentos cujo excesso apresentaria risco para a saúde, como gordura, sódio e açúcar. A variedade reflete o consumo de alimentos através dos cinco grupos principais. Nesse estudo existem dez variáveis (porcentagem de calorias fornecidas por gorduras totais, porcentagem de energia fornecida por gorduras saturadas, colesterol, porções de frutas, hortaliças, cereais, cálcio, ferro, diversidade e moderação). Cada variável adquire pontuação variando entre zero e 10 , sendo os maiores valores para os consumos ideais. Por fim, o escore máximo possível será $100^{13}$.

\section{A LI D A Ç Ã O E A PLICA B ILIDA DE}

Kant $(1996)^{2}$ realizou um trabalho de revisão dos índices dietéticos até então publicados. Essa revisão continha 59 trabalhos publicados entre as décadas de 40 e 90 , os quais apresentavam índices baseados no consumo de nutrientes, no consumo de alimentos ou grupos de alimentos e baseados no consumo de nutrientes e alimentos. Nesse trabalho, foi indicada a realização de validação para 52 índices em relação a alguns fatores, como para a ingestão de lipídios, adequação nutricional e ocorrência de doenças.

Dentre os vários índices analisados no presente trabalho, pôde-se verificar que a maioria não sofreu validação. Em geral, as pesquisas desenvolvidas para a aplicação dos índices foram realizadas com a função de se avaliar a qualidade da dieta de um grupo populacional específico. A aplicação dos índices não teve por objetivo analisar a sensibilidade destes para possíveis aplicações em estudos posteriores.

Somente no Índice de Qualidade da Dieta é notada a presença da validação. Após a aplicação do índice, os resultados indicaram, naquele momento, que este era um instrumento cuja análise da qualidade global da dieta fora bem sucedida por incorporar vários fatores relacionados a ela ${ }^{6}$. 
Assim, estudos sobre a validação dos vários índices da qualidade da dieta em contraposição ao estado nutricional identificado por parâmetros bioquímicos, antropométricos e clínicos ainda são necessários.

Mesmo assim, os índices dietéticos vêm sendo aplicados e sofrendo adaptações em outros estudos, visando, geralmente, comparar e avaliar a dieta de determinadas populações.

O Escore da Diversidade da Dieta, por exemplo, foi adaptado para ser um indicativo da adequação dietética de crianças entre dois e três anos em um estudo realizado nos Estados Unidos. Desse modo, ocorreram alterações tanto no número como no tamanho das porções dos grupos de alimentos ${ }^{3}$.

No Brasil, em um estudo realizado com idosas, o Índice de Alimentação Saudável (IAS) sofreu adaptações tanto no método de aplicação dos inquéritos alimentares como na composição do índice. Ele passou a ter oito itens, sendo eles os grupos de alimentos do Guia da Pirâmide Alimentar Adaptada ${ }^{16}$, que modificou a pontuação total do índice ${ }^{17}$.

Baseando-se nos conceitos do Índice de Qualidade da Dieta (IQD), foi elaborado, recentemente, um índice adaptado à população chinesa. Apesar de possuir os mesmos princípios do IQD original, esse índice foi desenvolvido a partir dos guias dietéticos para a população chinesa. Assim, foram feitas adaptações com relação aos componentes do índice e aos parâmetros de distribuição de pontos. Também foi dada importância em se avaliar tanto a deficiência como o excesso nutricional, pois ambas as situações são bastante evidentes naquele país. Esse índice foi validado através da verificação de sua eficácia em relação às expectativas criadas, anteriormente, dos resultados que o índice deveria apresentar a respeito da população chinesa. Por meio desse estudo, concluiu-se que o índice capta a variação da qualidade da dieta através de seus componentes e é sensível em identificar tanto a deficiência como 0 excesso nutricional ${ }^{18}$.
VANTAGENSE DES VANTAG N S DE CADA ÍNDICE

Analisando o índice de nutrientes, é válida a avaliação global da dieta através da análise do consumo de alguns nutrientes, os quais podem predizer o consumo de muitos outros. Entretanto, é importante frisar que a qualidade da dieta está relacionada com a restrição na ingestão de algumas substâncias, como sódio, colesterol e gordura saturada, além de se relacionar com a ingestão adequada de muitos nutrientes. Pelo fato de o índice não avaliar esses outros fatores, a análise dietética realizada é incompleta.

O tamanho da amostra utilizada no estudo do EVD é menor, em comparação com trabalhos apresentados, mas ele se destaca pela indicação de uma abordagem diferente da análise por nutriente: a variedade. Esta variedade é, indubitavelmente, importante indicador da qualidade da dieta, pois uma alimentação com maior número de itens tende a alcançar a adequação para todos os nutrientes. Porém, essa afirmação nem sempre pode ser tida como verdadeira, como também concluiram McCrory et al. (1999) ${ }^{19}$. O simples consumo de diferentes alimentos não indica que foram consumidos alimentos de to dos os grupos. Assim, analisando a dieta somente através do escore da variedade da dieta, os resultados obtidos podem não refletir a realidade.

No tocante ao escore da diversidade da dieta, não são mencionadas em suas análises as ingestões de bebidas alcoólicas, gorduras, doces e, tampouco, o sódio ou colesterol. Assim, apesar de o escore fornecer a qualidade da dieta em relação às recomendações para os cinco grupos alimentares feitas pela Pirâmide, ele deixa de fornecer informações sobre alguns indicadores que podem afetar o resultado final da avaliação da dieta.

Pressupõe-se que a escolha da análise por grupos de alimentos em vez de alimentos individuais, no EDD, denuncie limitações no consumo de alguns grupos de alimentos ${ }^{6}$. 
O EVD conta todos os alimentos consumidos, incluindo condimentos. Sozinho, pode dar uma falsa impressão favorável da qualidade da dieta. No entanto, um alto EDD reflete um consumo de alimentos de diversos grupos.

O escore da diversidade da dieta e o escore da variedade da dieta foram usados conjuntamente em um trabalho realizado por Hatloy et al. (1998) ${ }^{4}$, em Mali, África, e pôde-se observar através desse que o EDD é um melhor indicador da dieta, em comparação com uma simples contagem de alimentos, feita pelo EVD. Entretanto, tais escores proporcionam simples indicações para serem usadas em larga escala, em estudos demográficos e nutricionais. Assim, apesar de o EDD e o EVD não darem uma completa visão da ingestão adequada de nutrientes, os resultados da pesquisa mostraram que esses escores podem fornecer boa indicação da adequação nutricional da dieta, em particular, quando combinados.

Em relação ao índice de qualidade da dieta, sua avaliação é feita de modo a obter uma visão global desta. Porém, o método de distribuição de pontos pode gerar dificuldades na interpretação dos resultados. Estas dificuldades prendem-se ao fato de a dieta adequada receber pontuação igual ou próxima a zero, enquanto a inadequada receberia maior quantidade de pontos.

O índice de alimentação saudável se baseia nas mais recentes recomendações dietéticas publicadas. Portanto, é bastante possível a obtenção de informações para classificar a qualidade da dieta em má ou boa, de forma resumida e simples. Porém, tal índice não faz menção sobre o consumo de álcool, que poderia ser outro importante fator na análise da dieta.

0 índice de qualidade da dieta revisado forneceu resultados mais globais, já que novos indicadores foram incluídos na análise. Esse índice proporciona uma conclusão mais abrangente por avaliar aspectos distintos e compactá-los em um só resultado.

Como foi apresentado por Haines et al. (1999) ${ }^{13}$, o índice de qualidade da dieta revisado captura com sucesso a qualidade global da dieta por refletir a variação de componentes individuais nos quais o índice é baseado e por incluir o consumo de substâncias como fibras e vitaminas, que não são variáveis do índice. 0 uso de medidas simples da dieta, como a porcentagem de energia fornecida pelas gorduras, não explica, totalmente, a complexidade desta, como o IQD-R o faz.

0 índice de qualidade da dieta revisado e o índice de alimentação saudável (IAS) possuem semelhanças e diferenças. Ambos incorporam nutrientes e alimentos como componentes e estão baseados em uma escala de 100 pontos. Os dois incluem recomendações estimadas para porções de frutas, hortaliças e cereais. O IAS também mede porções de carne e leite, enquanto o IQD-R mede a adequação para ferro e cálcio. Os dois possuem porcentagem de energia proveniente de gorduras totais e saturadas e colesterol dietético. A pesar de ambos incluírem medidas de variedade, as definições deste termo diferem. O IQD-R inclui a variável moderação, enquanto o IAS analisa 0 consumo de sódio, somente. Por último, o IAS destina-se a pessoas com dois anos ou mais, enquanto o IQD-R destina-se a indivíduos maiores de 18 anos $^{13}$.

Atualmente, as recomendações para diversos nutrientes são baseadas no seu consumo em determinada amostra da população. No entanto, isso não implica que as recomendações para esses nutrientes satisfaçam as necessidades nutricionais de um indivíduo. O IQD-R realiza a avaliação da dieta baseando-se, algumas vezes, no consumo bruto de determinados nutrientes, como o cálcio. Este mineral, segundo as novas Ingestões Dietéticas de Referência (Dietary Reference Intakes - DRI), tem como parâmetro de referência a Ingestão Adequada (AI), a qual não deve ser utilizada para avaliar adequação de consumo ${ }^{20,21}$. Assim, para o uso do IQD-R, os parâmetros de avaliação da adequação para cálcio deverão ser as recomendações anteriore ${ }^{22}$ ou nova adaptação deverá ser elaborada.

Já o IAS baseia-se, principalmente, na avaliação através do consumo de porções de 
grupos de alimentos. Tal fato coloca este índice em situação vantajosa por fornecer resultados mais aplicáveis na avaliação dietética para alguns nutrientes.

A avaliação global da dieta, sendo resumida em uma única variável em substituição da análise dietética decomposta em várias subunidades, surge como uma nova ferramenta para proporcionar melhor avaliação da dieta em estudos epidemiológicos, já que esta terá maior representatividade ${ }^{2,10}$. A escolha de um índice, em particular, depende das características da população a ser analisada, do método usado e dos recursos disponíveis para a análise dos dados dietéticos². Por isso, segundo Stookey et al. $(2000)^{18}$, mais estudos serão necessários para o desenvolvimento de índices aplicáveis em grupos populacionais.

\section{COMENTÁRIOS FINAIS}

Considerando a população brasileira e tendo em vista que os índices existentes têm outras procedências, como a americana, inglesa ou francesa, não existe um índice específico para avaliá-la. As características dos brasileiros podem diferir, pois as condições socioeconômicas e de hábitos alimentares aqui encontradas não são as mesmas dos países desenvolvidos. Um estudo selecionando uma amostra representativa da população brasileira e avaliando os fatores, nutricionais ou não, como medidas antropométricas e estilo de vida, sem deixar de lado a escolha do método mais adequado para coleta de dados, é válido para o conhecimento das necessidades e características da população, já que fornecerá resultados simplificados da qualidade da dieta. Tais resultados são importantes e serão úteis em diversas outras áreas relacionadas com a Saúde Pública.

Vale ressaltar a necessidade de avaliar a inclusão de medidas globais ou relativas da energia da dieta como, por exemplo, a proporção energética originária de carboidratos. Para o índice poder refletir melhor as orientações dietéticas fundamentais, baseadas na proporcionalidade, variedade e moderação, ele não deve estar relacionado com quantidades absolutas de alimentos consumidos.

Pela análise das utilidades dos índices, pode-se concluir que esses possuem fundamental importância ao indicar os diferentes componentes da dieta, fornecendo um resultado que é, ao mesmo tempo, global e simplificado, quando comparado às análises realizadas a partir da quantificação de todos os nutrientes citados nas recomendações nutricionais.

Entretanto, novos estudos ainda são necessários para a obtenção de resultados concretos e conseqüente elaboração de índices abrangentes e aplicáveis.

\section{REFERÊ N CIAS}

1. Wolfe WS, Campbell CC. Food pattern, diet quality, and related characteristics of schoolchildren in New York State. J Am Diet Assoc 1993; 93:1280-4

2. Kant AK. Indexes of overall diet quality: a review. J Am Diet Assoc 1996; 96(8):785-91.

3. CoxDR, Skinner JD, Carruth BR, Moran III J., Houck KS. A food variety index for toddlers (VIT): development and application. J Am Diet Assoc 1997; 97(12):1382-88.

4. Hatloy A, Torheim LE, Oshaug A. Food variety: a good indicator of nutritional adequacy of diet? A case study from an urban area in Mali, West of Africa. Eur J Clin Nutr 1998; 52:891-8.

5. Kant AK, Schatzkin A, Graubard BI, Schairer C. A prospective study of diet quality and mortality in women. J Am Med Assoc 2000; 283(16): 2109-15.

6. Patterson RE, Haines PS, Popkin BM. Diet quality index: capturing a multidimensional behavior. J Am Diet Assoc 1994; 94(1):57-64.

7. Drewnowski A, Henderson AS, Driscoll A, Rolls BJ . The dietary variety score: assessing diet quality in healthy young and older adults. J Am Diet Assoc 1997; 97(3):266-71. 
8. Jenkins RM, Guthrie HA. Identification of index nutrients for dietary assessment. J Nutri Educ 1984; 16(1):15-8.

9. Krebs-Smith SM, Smiciklas-Wright H, Guthrie HA, Krebs-Smith J. The effects of variety in food choices on dietary quality. J Am Diet Assoc 1987; 87(7):897903.

10. Drewnowski A, Henderson AS, Shore AB, Fischler $C$, Preziosi $P$, Hercberg $S$. Diet quality and dietary diversity in France: implications for the French paradox. J Am Diet Assoc 1996; 96(7):663-9.

11. United States Department of Agriculture. Dietary Guidelines for Americans [online]. Washington; 2001a [cited 2001 Feb 27]. Available from: URL: http://www.nalusda.gov/fnic/dga/dguide95.html

12. United States Department of Agriculture. The Food Guide Pyramid [online]. Washington; 2001b [cited 2001 Feb 27]. Available from: URL: http:// www.nalusda.gov/fnic/dga/dga95.html

13. Haines PS, Siega-Riz AM, Popkin BM. The diet quality index revised: a measurement instrument for populations. J Am Diet Assoc 1999; 99(6): 697-704.

14. Kennedy ET, Ohls J, Carlson S, Fleming K. The healthy eating index: design and applications. J Am Diet Assoc 1995; 95(10):1103-8.

15. Bowman AS, Lino M, Gerrior AS, Basiotis PP. The healthy eating index: 1994-96. Washington DC: Department of Agriculture; 1998.
16. Philippi ST, Latterza AR, Cruz AT, Ribeiro LC. Pirâmide alimentar adaptada: guia para a escolha dos alimentos. Rev Nutr 1999; 12(1):65-80.

17. Brasil BG. Do outro lado do muro: percepções de idosas institucionalizadas sobre a alimentação [tese]. São Paulo: Universidade de São Paulo; 2001.

18. Stookey JD, Wang Y, Ge K, Lin H, Popkin BM. Measuring diet quality in China: the INFH-UNC-CH diet quality index. Eur J Clin Nutr 2000; 54(11): 811-21.

19. M ccrory MA, FUSS PJ, McCallum JE, YAO M, VINKEN AG, HAYS NP, et al. Dietary variety within food groups: association with energy intake and body fatness in men and women. Am J Clin Nutr 1999 69:440-7.

20. Food and nutrition board. Dietary reference intakes: applications in dietary assessment. [on line] Washington DC: National Academy Press; 2000 [cited 2001 Oct 12] Available from: URL: http:// www.nap.edu

21. Simpósio Brasileiro de Alimentação e Nutrição. Usos e aplicações das "dietary reference intakes" DRIs [CD-ROM]. São Paulo; 2001.

22. National Research Council (USA). Recommended dietary allowances. 10th. Washington DC: National Academy Press; 1989.

Recebido para publicação em 16 de maio de 2001 e aceito em 23 de setembro de 2002 